\title{
Editorial
}

\section{Categorification in Representation Theory}

\author{
Alistair Savage, ${ }^{1}$ Aaron Lauda, $^{2}$ and Anthony Licata ${ }^{3}$ \\ ${ }^{1}$ University of Ottawa, Canada \\ ${ }^{2}$ Columbia University, USA \\ ${ }^{3}$ Stanford University, USA \\ Correspondence should be addressed to Alistair Savage, alistair.savage@uottawa.ca
}

Received 31 December 2010; Accepted 31 December 2010

Copyright ( 2010 Alistair Savage et al. This is an open access article distributed under the Creative Commons Attribution License, which permits unrestricted use, distribution, and reproduction in any medium, provided the original work is properly cited.

Categorification, a term coined by Louis Crane and Igor Frenkel, is the process of realizing mathematical structures as shadows of higher mathematics. The original motivation was the idea that one should be able to construct four-dimensional quantum field theories by categorifying the representation theory of quantum groups. Work of many people over the past 15 years has made great progress in clarifying and developing this vision. In that time, it has become increasingly clear that categorification is a broad mathematical phenomenon with applications extending far beyond these original motivations.

This special issue focuses on categorification in the context of representation theory of quantum groups and Hecke algebras, in the spirit of the examples of categorification coming from geometric representation theory and low-dimensional topology. A special emphasis is placed on the diagrammatic calculus created by Elias and Khovanov to describe the category of Seorgel bimodules in terms of planar diagrams.

One reason for the prominence of quantum groups and Hecke algebras in categorification is that they provide a bridge between representation theory and low-dimensional topology. Indeed, quantum groups and Hecke algebras can be viewed as the basic algebraic input giving rise to knot invariants such as the Jones polynomial, colored Jones polynomial, HOMFLYPT polynomial, and Witten-Reshetikhin-Turaev 3-manifold invariants. Khovanov showed that the Jones polynomial could be understood as the graded Euler characteristic of a graded homology theory for knots and links, thus opening the door to a new chapter of interaction between representation theory and low-dimensional topology. Besides being a strictly stronger knot invariant than its Euler characteristic, many of the knot homologies developed since Khovanov homology are functorial: cobordisms between tangles give rise to maps between homologies. This functoriality is explained by the rich structure of morphisms and natural transformations which exists in categorified quantum groups and Hecke algebras. 
That representation theory has proven to be an especially fertile ground for categorification is a fact that owes much to the geometric methods pervading the subject. For example, constructions of natural bases with positivity and integrality properties are a central part of geometric representation theory. The categorifications that sit above such geometric constructions provide rich explanations for the existence of these bases: in the categorification, basis vectors are reinterpreted as indecomposable objects in a category, while structure constants become decomposition numbers or multiplicities. From this point of view, positivity and integrality are manifest. A particularly important object in geometric representation theory is the category of Soergel bimodules, which was used by Soergel to give a categorification of the Hecke algebra. The Elias-Khovanov description of the Soergel category also suggests a natural categorification of a quotient of the Hecke algebra known as the Temperley-Lieb algebra. Such a categorification is studied in the papers in this issue by B. Elias and B. Elias-Khovanov.

Constructions of knot homology theories associated to quantum $\mathfrak{s l}_{n}$ can be given using certain singular surfaces called foams. The papers by P. Vaz and M. Mackaay-P. Vaz in this issue construct representations of the Soergel category on the category of foams. These constructions clarify the representation theoretic meaning of foams in the construction of $\mathfrak{s l}_{n}$ knot homology theories. D. Krasner, in his paper, constructs knot homology from braid group actions built from the diagrammatic Soergel category, obtaining integral versions of HOMFLY-PT and $\mathfrak{s l}_{n}$-link homology theories.

J. Sussan and D. Hill's paper connects the Khovanov-Lauda diagrammatic categorification of $U_{q}\left(\mathfrak{s l}_{n}\right)$ with a previous categorification of the adjoint representation constructed by Khovanov-Huerfano. In particular, they obtain a categorification of the irreducible representations of highest weight $2 \omega_{k}$, where the $\omega_{k}$ are the fundamental weights. The paper by A. Ram and P. Tingley uses the Shapovalov determinant for a universal Verma module to explain a connection between structure constants appearing in the Misra-Miwa Fock space and Weyl modules.

One theme common to several of the papers in this special issue is the use of a graphical calculus of planar diagrams in categorification. In these graphical presentations, the algebraic relations amongst morphisms are described by local geometry in the plane. While these diagrammatic constructions can be rigorously translated into a purely algebraic formulations, planar diagrams describe categorifications in a geometric and often quite intuitive manner.

Alistair Savage

Aaron Lauda

Anthony Licata 


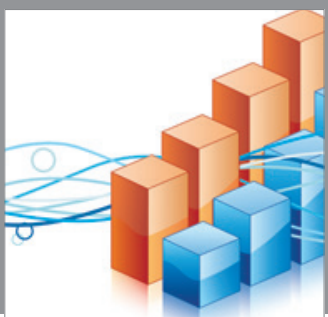

Advances in

Operations Research

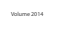

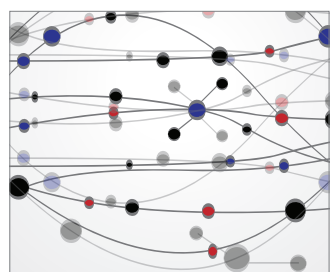

\section{The Scientific} World Journal
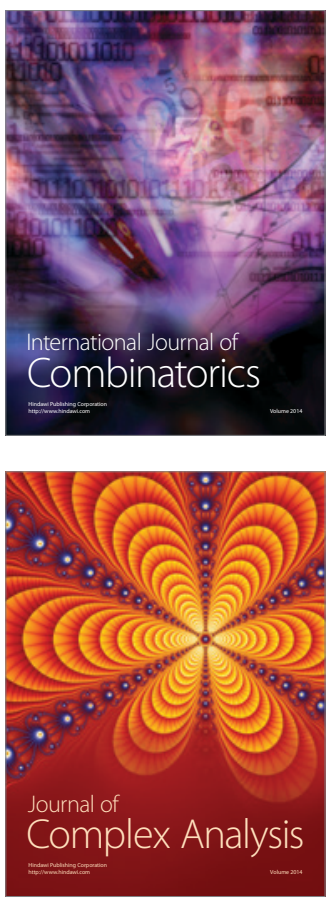

International Journal of

Mathematics and

Mathematical

Sciences
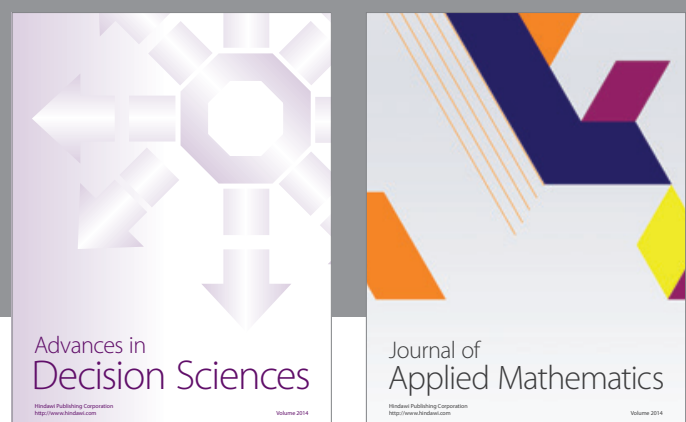

Journal of

Applied Mathematics
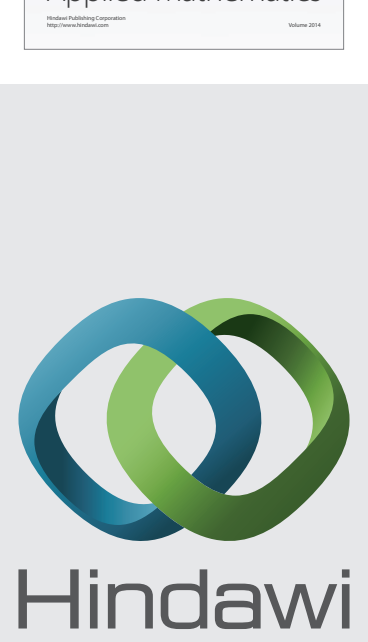

Submit your manuscripts at http://www.hindawi.com
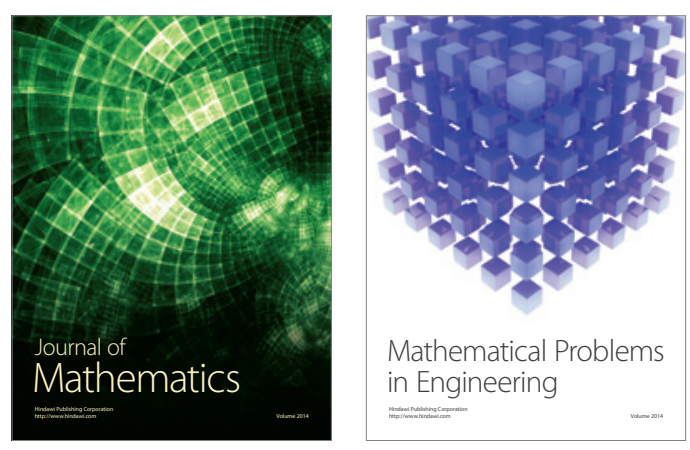

Mathematical Problems in Engineering
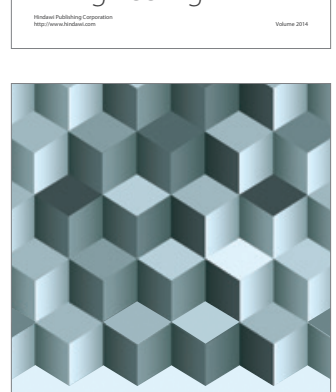

Journal of

Function Spaces
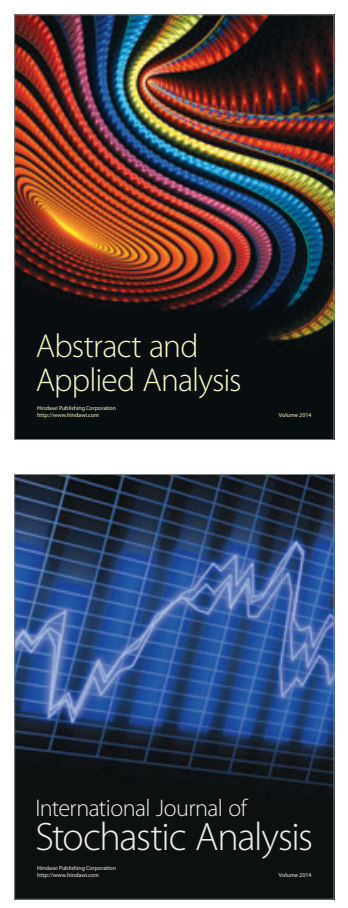

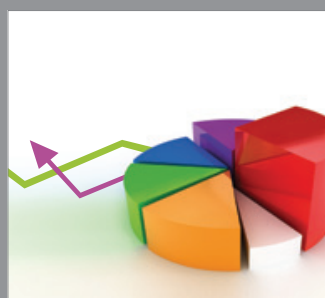

ournal of

Probability and Statistics

Promensencen
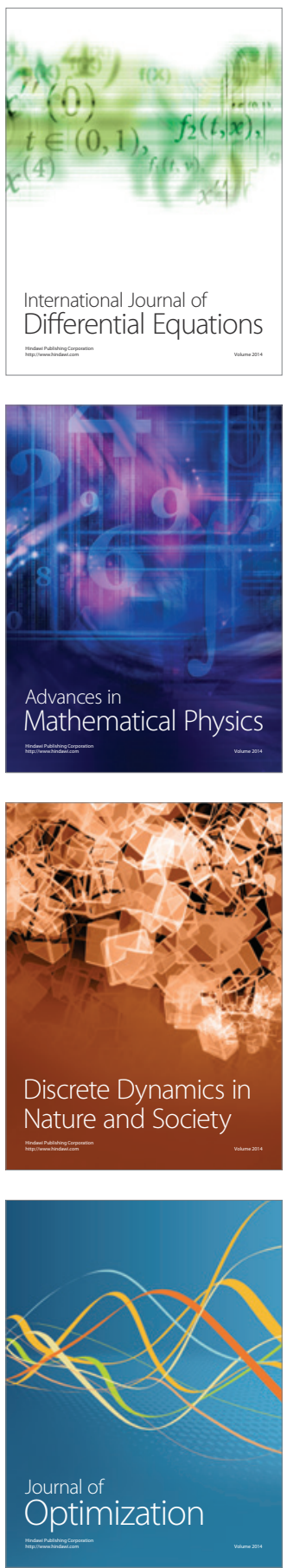